\title{
Steps to Establish International Radio Quiet Zones
}

\author{
Harvey Butcher \\ Netherlands Foundation for Research in Astronomy, \\ P.O. Box 2, 7990 AA Dwingeloo, The Netherlands
}

\begin{abstract}
Future radio telescopes will feature large increases in sensitivity, not only to celestial sources but also to man-made interference. In addition, they will need to measure spectral lines as a function of redshift and hence observe at arbitrary frequencies. Current international regulations governing the use of the radio spectrum are in principle inappropriate to these science-driven needs. There are still a few places on Earth that are relatively interference-free, but the coming generation of telecommunications satellites in low orbit will compromise even those sites. The Organization for Economic Cooperation and Development (OECD) has recently sponsored a working group on the future of radio astronomy, which recommended that the possibility of establishing one or more formally recognized International Radio Quiet Zones (IRQZ) be studied seriously. First results of that effort will be discussed.
\end{abstract}

